\title{
Percutaneous endoscopic gastrostomy (PEG) using a novel large-caliber introducer technique kit: a retrospective analysis
}

Authors

Institutions

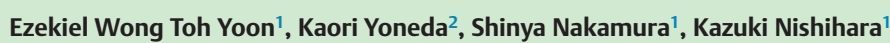

${ }^{1}$ Department of Internal Medicine (Gastroenterology), Hiroshima Kyoritsu Hospital, Hiroshima City, Japan

2 Endocopy Center, Hiroshima Kyoritsu Hospital, Hiroshima City, Japan submitted 18. April 2016 accepted after revision 6. July 2016

\section{Bibliography}

Dol http://dx.doi.org/

10.1055/s-0042-112587

Published online: 30.8.2016

Endoscopy International Open 2016; 04: E990-E996

(c) Georg Thieme Verlag KG

Stuttgart · New York

E-ISSN 2196-9736

\section{Corresponding author}

Ezekiel Wong Toh Yoon, MD

Department of Internal

Medicine

Hiroshima Kyoritsu Hospital

2-20-20 Nakasu Asaminami-ku

Hiroshima City

Japan

Phone: +81-82-879-1111

easybs@hotmail.com

\section{License terms}

(ㅇ) $\Theta \circledast$
Background and study aims: Percutaneous endoscopic gastrostomy (PEG) using the introducer technique is not only useful in patients with upper digestive tract stenosis but has been shown to reduce peristomal infection. In this study, we evaluated the safety and utility of a novel largecaliber introducer PEG kit (using 20 Fr size tube) compared with a push kit of similar size.

Patients and methods: One hundred and thirtysix patients who received PEG at our hospital between January 2014 and December 2015 were retrospectively analyzed. Baseline characteristics, laboratory biomarkers, hemodynamic changes, postoperative adverse events and clinical outcomes with both kits were compared.

Results: The new introducer PEG kit was used in 61 patients while the remaining 75 patients received tube placement using a push technique

\section{Introduction}

\section{$\nabla$}

Percutaneous endoscopic gastrostomy (PEG) is a safe, minimally invasive procedure for long-term enteral nutrition in patients with impaired oral intake [ $1-3]$. However, aseptic procedure is difficult to maintain in the pull or push technique with the probable transfer of oral bacterial to the stoma site. Peristomal site infection has often been identified as the most common adverse event after PEG tube placements [4]. Administration of systemic prophylactic antibiotics has been demonstrated to be effective in reducing peristomal infection and is often employed before the procedure [5-7]. Nonetheless, fungal infection may still present a problem in the severely ill or immunocompromised patient [8].

PEG using the introducer technique, in which the gastrostomy tube does not pass through the oral cavity during placement, was first described by Hashiba in 1980 [9]. It was also independently developed and described by Inoue and Russell later on in 1983 and 1984 respectively [10,11]. This
PEG kit. Except for the prevalence of dementia, which was lower in the introducer PEG kit group, baseline characteristics were similar in both groups. Tube placements were $100 \%$ successful with both PEG kits and there were no significant differences in the change of postoperative hemodynamic or laboratory biomarkers. The Introducer PEG kit group experienced fewer incidence of feeding-related aspiration pneumonia (8.2\% vs. $24 \%, P=0.02$ ), lower peristomal infection scores (1.2 vs. $1.6, P<0.01$ ), shorter postoperative length of stay (16 days vs. 23.7 days, $P=0.01$ ) and fewer deaths at day $60(3.3 \%$ vs. $16 \%, P=0.02)$.

Conclusions: Gastrostomy using the new largecaliber introducer PEG kit is safe and produced non-inferior (with some favourable) results when compared to the push technique using similar size tubes.

technique is extremely useful in patients with difficulty in opening their mouth or stenosis of the upper digestive tract such as head and neck cancer patients [12]. While the pull or push techniques may not be feasible in up to $20 \%$ of these patients, the introducer technique using an ultrathin endoscope transnasally not only increases the success rate of tube placement but also decreases the probability of tumor seeding during procedure $[13,14]$. Furthermore, because the gastrostomy tube is inserted directly via the abdomen during placement, rates of peristomal site infection have been shown to be reduced in previous studies, including a recent meta-analysis $[15,16]$. One study even concluded that the introducer technique can be performed without the need of prophylactic antibiotic administration [17].

Although the safety of the introducer technique was greatly enhanced with the introduction of endoscopic gastropexy, one of the limitations of this technique was that almost all commercially available kits used small-caliber gastrostomy 
tubes of up to 15 Fr size, which may be associated with a higher risk of tube obstruction $[18,19]$. In this study, we evaluated the safety and utility of a recently available large-caliber (20 Fr) introducer PEG kit with an improved puncture needle system compared to a similar size push technique kit used in our hospital.

\section{Patients and methods \\ $\nabla$}

\section{Study design and patients}

Prospectively collected data from patients who received PEG at our hospital between January 2014 and December 2015 was analyzed retrospectively. Only patients receiving tube placement for enteral feeding were included and after excluding 3 patients (1 patient because tube placement was for gastric decompression and 2 patients because of insufficient data), 136 patients (64 males and 72 females) were enrolled into this study. Data regarding baseline characteristics such as age, gender, comorbidities, and preoperative biomarkers (body mass index and blood laboratory markers collected on day of procedure) as well as postoperative findings such as hemodynamic changes immediately after procedure, changes in laboratory data on Day 7 after the procedure, postoperative adverse events (peristomal site infection, feeding-related pneumonia etc.) and clinical outcomes of interest (postoperative length of stay and mortality) were compared according to the different PEG techniques used. This study protocol was reviewed and approved by the ethics review committee of Hiroshima Kyoritsu Hospital. All patients, or their legal guardians, provided written informed consent for the procedures as well as enrolment into the study.

\section{PEG tube insertion procedures}

All procedures were performed in our endoscopy center by at least 2 experienced gastroenterologists. Preoperative and postoperative protocols employed were the same no matter which insertion technique was used. Prophylactic antibiotics were administered in all patients (as required by existing protocols) but the use of conscious sedation with diazepam was left to the discretion of endoscopists, with careful monitoring of hemodynamic changes at all times during the procedure. Patients with antithrombotic therapy (using antiplatelet drugs or anticoagulants) were managed according to the latest guidelines established by the Japan Gastroenterological Endoscopy Society.

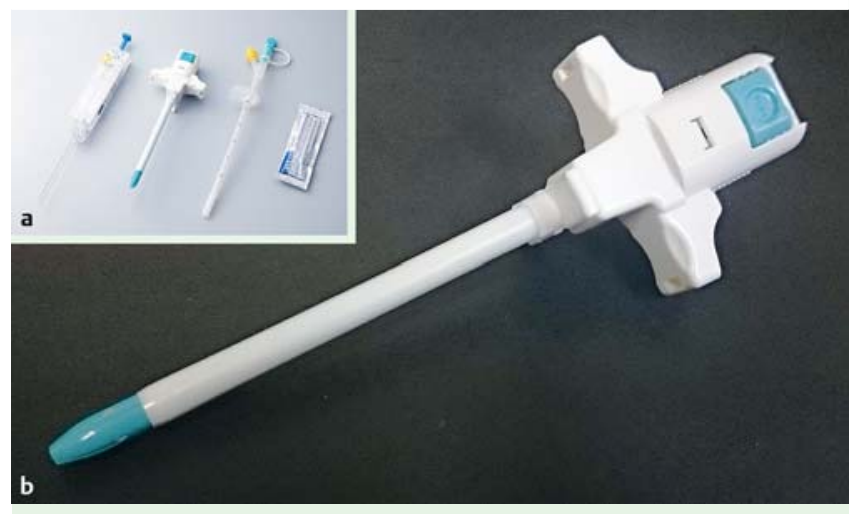

Fig. 1 a New introducer PEG kit with $\mathbf{b}$ specialized puncture needle/trocar enlarged.

In the push technique, a 20 Fr tube (Safety PEG kit, Boston Scientific Corp., USA) is passed over a guidewire through the mouth into the stomach. This procedure is performed without gastropexy. It should be noted that although some previous studies have mistakenly used the term "push" method when they actually refer to the introducer technique, the push technique in this study refers to the technique originally described by Sacks and Vine in 1983 [20]. For the introducer technique, a recently developed kit ( Fig. 1a, Intolief PEG kit by Create Medic Co., Ltd, Yokohama, Japan) using a 20 Fr gastrostomy tube was used. This kit includes a Funada-style gastropexy device as well as a puncture needle/trocar ( $\bullet$ Fig. 1b) with a newly developed sleeve protection system to avoid mispuncture of the opposite stomach wall.

- Fig. 2 is a pictorial diagram of the sleeve protection system during insertion of the puncture needle/trocar. The puncture needle is initially hidden under a protection sleeve before insertion ( $\nabla$ Fig. 2a). The penetrating edge of the puncture needle is exposed from the protection sleeve during insertion ( $\bullet$ Fig. $\mathbf{2 b}$ ) but once resistance from the abdominal and gastric walls is absent, the protection sleeve covers the puncture needle, locking it in as the external peel-away sheath enters the gastric lumen ( $\bullet$ Fig. 2c). This not only helps avoid any accidental puncture into the opposing gastric wall but also decreases the risk of needle-stick injury in medical personnel.

- Fig. 3 shows endoscopic imaging of PEG tube insertion with the introducer technique kit in a 79-year-old female under mechanical ventilation. After determining and sterilizing the puncture
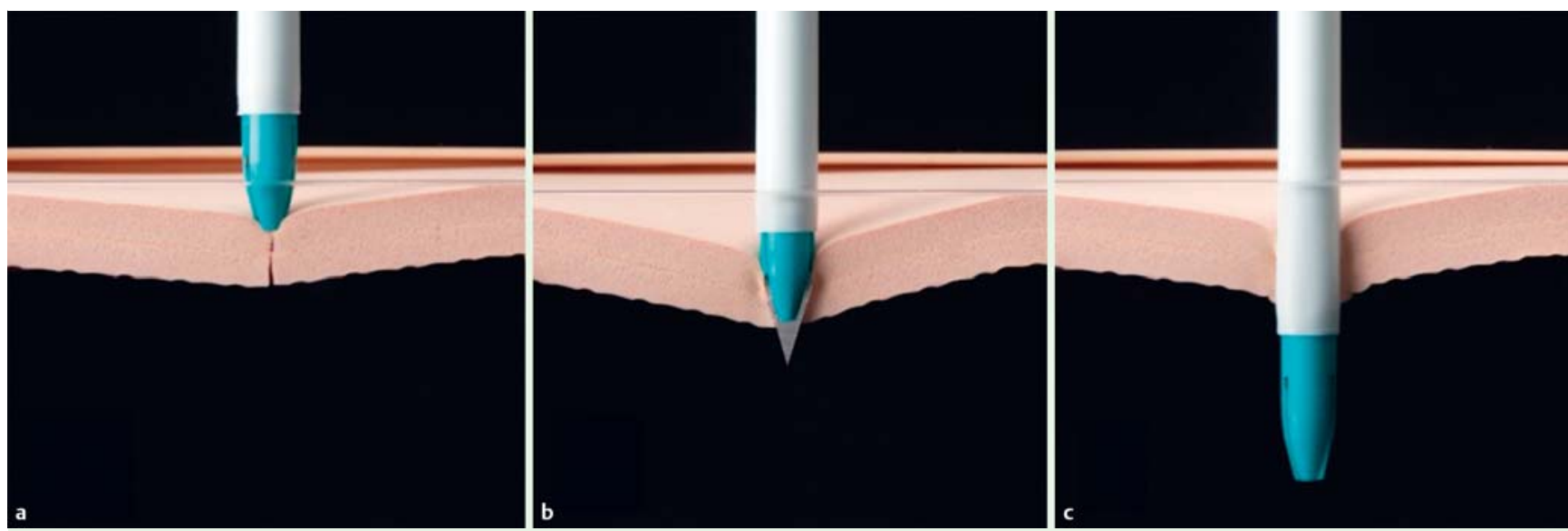

Fig. 2 Pictorial diagram showing insertion of puncture needle with protection sleeve. 

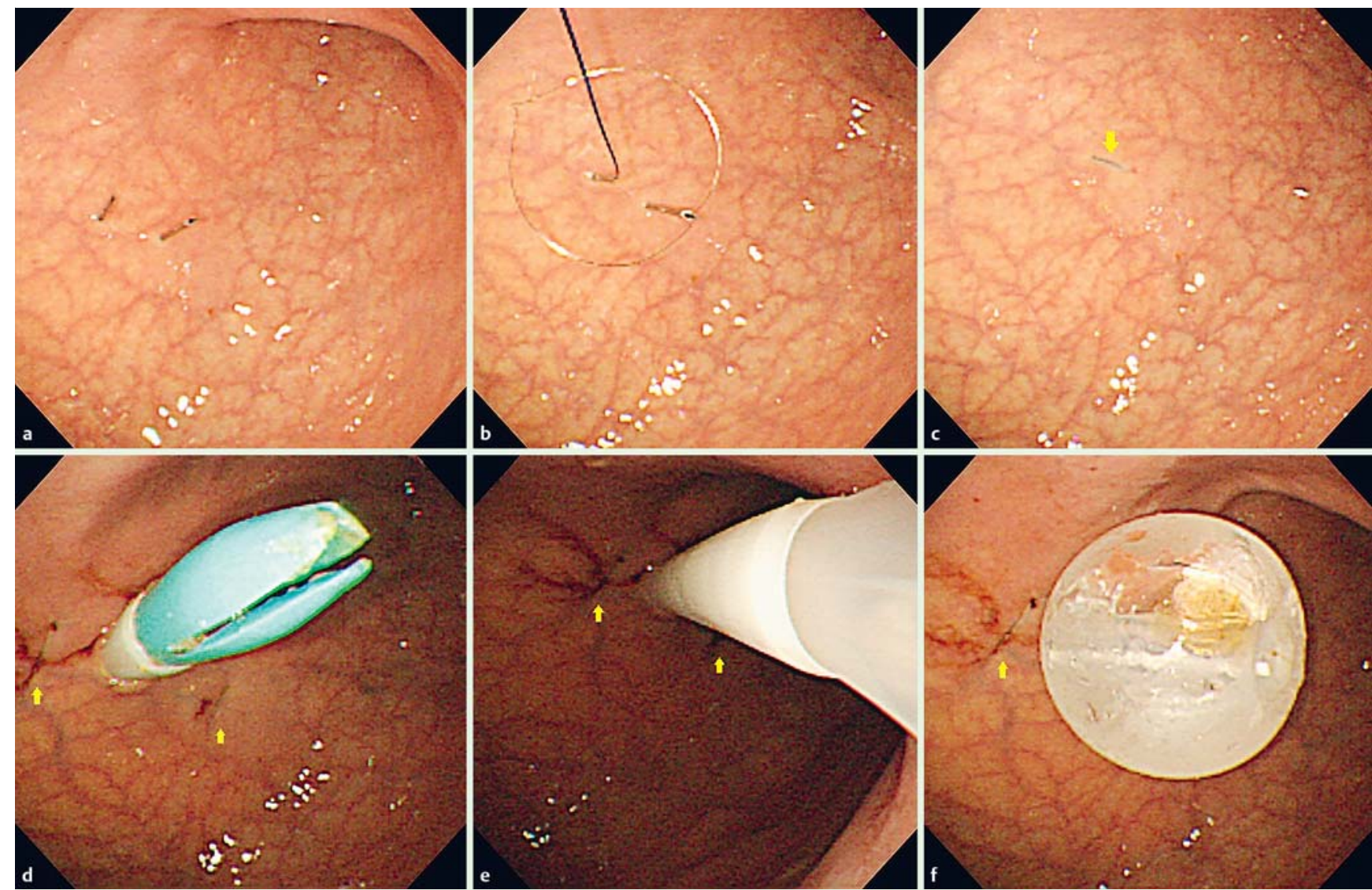

Fig.3 Endoscopic imaging of tube insertion using the new introducer PEG kit with yellow arrows marking the visible gastropexy sutures.

site, local anesthesia was applied and gastropexy performed. First, the double needle of the gastropexy device was inserted into the gastric lumen ( $\bullet$ Fig.3a). After a loop snare was formed from the tip of one needle, a suture thread was inserted via the other ( $\bullet$ Fig.3b). The suture thread was then caught by closing the loop snare and after the double needle was withdrawn from the stomach, the thread was tied outside the abdominal wall ( $\bullet$ Fig. 3c). This procedure was repeated at least 3 times surrounding the intended puncture site in a triangular pattern with a minimum distance of $2.0 \mathrm{~cm}$ apart in order to achieve stable gastropexy. After a $10 \mathrm{~mm}$ external incision was performed on the intended puncture site, the puncture needle/trocar $(6.7 \mathrm{~mm}$ or $20 \mathrm{Fr}$ ) was then inserted ( $\mathrm{Fig} . \mathbf{3 d}$ ). Leaving only the external peel-away sheath, the puncture needle was removed and a $20 \mathrm{Fr}$ gastrostomy tube was inserted via the sheath $($ Fig.3e). After inflating the retention balloon with $10 \mathrm{~mL}$ of distilled water, the external sheath was peeled away to leave only the PEG tube in place (ه Fig.3f).

Although an ultrathin endoscope can be used transnasally for the introducer technique, procedures are usually performed by oral endoscopy. The choice of PEG kit is determined by the operator, influenced by preference or technical skills. Nonetheless, use of the introducer PEG kit is indicated in patients who cannot open their mouth and strongly recommended in cases where single endoscope insertions are thought to be preferable, such as patients with mechanical ventilation. PEG feeding usually begins 2 days after the procedure (using standard protocol) and patients are followed up by our nutrition support team where peristomal sites are also evaluated after 7 days. Peristomal infection was assessed using a previously validated scoring system (Jain's infec- tion score) by assigning a score for erythema (0 to 4$)$, induration ( 0 to 3 ) and exudate ( 0 to 4 ) [21]. Infection was defined as development of pus or a combined infection score of 8 or more. Gastropexy sutures were kept in for at least 2 weeks after the procedure.

\section{Statistical analysis}

Continuous variables are expressed as mean (standard deviation with range occasionally). Categorical (qualitative) variables are expressed as numbers (percentage). Comparisons for continuous variables were made using the Student $t$-test for normal data and the Mann-Whitney U test for non-parametric data. Tests for proportionality between groups were made using the chi-square test (or Fisher's exact test when indicated). Statistical significance was defined as $P<0.05$ and analysis was performed using XLSTAT2014 for Windows (Addinsoft Ltd., Paris, France).

\section{Results}

$\nabla$

In total, 136 patients were enrolled in the study. Seventy-five patients received tube placement with PEG kits using the push technique (Push kit) and 61 patients received placement with the new introducer PEG kit (Introducer kit). Baseline characteristics of patients (age, gender, comorbidities and preoperative biomarkers) are shown in Table 1 . The patients ranged in age from 59 years to 97 years and there were no significant differences in age or gender between the two groups. For comorbidities, although there were no differences in prevalence of stroke, neurodegenerative disorders, respiratory disorders, cardiovascular dis- 
Table 1 Patient characteristics.

\begin{tabular}{|c|c|c|c|}
\hline & $\begin{array}{l}\text { Push kit } \\
(n=75)\end{array}$ & $\begin{array}{l}\text { Introducer kit } \\
(n=61)\end{array}$ & $P$ value \\
\hline Age, years, mean (SD, range) & $81.5(9.7,60-97)$ & $81.6(7.7,59-95)$ & 0.64 \\
\hline Gender (male/female) & $34 / 41$ & $30 / 31$ & 0.73 \\
\hline \multicolumn{4}{|l|}{ Comorbidities } \\
\hline Stroke, $n(\%)$ & $59(78.7)$ & $44(72.1)$ & 0.42 \\
\hline Antithrombotic therapy, $n(\%)$ & $29(38.7)$ & $17(27.9)$ & 0.21 \\
\hline Dementia, $n(\%)$ & $33(44)$ & $13(21.3)$ & $<0.01$ \\
\hline Neurodegenerative disorders, $n(\%)$ & $14(18.7)$ & $9(14.8)$ & 0.65 \\
\hline Respiratory disorders, $n(\%)$ & $40(53.3)$ & $26(42.6)$ & 0.23 \\
\hline Cardiovascular disorders, $n(\%)$ & $24(32)$ & $23(37.7)$ & 0.59 \\
\hline Diabetes mellitus, $n$ (\%) & $16(21.3)$ & $19(31.1)$ & 0.24 \\
\hline Malignancy, $n(\%)$ & $9(12)$ & $8(13.1)$ & 0.84 \\
\hline \multicolumn{4}{|l|}{ Preoperative biomarkers } \\
\hline Body mass index, $\mathrm{kg} / \mathrm{m}^{2}$, mean (SD) & $18.0(3.5)$ & $17.8(3.0)$ & 0.98 \\
\hline White blood count, / $\mu \mathrm{L}$, mean (SD) & $7627(2844)$ & $7016(2296)$ & 0.27 \\
\hline Hemoglobin, g/dL, mean (SD) & $11.3(1.8)$ & $11.4(1.9)$ & 0.52 \\
\hline Platelet count, $\times 10^{4} / \mu \mathrm{L}$, mean (SD) & $26.6(9.8)$ & $25.6(8.9)$ & 0.56 \\
\hline Serum albumin, g/dL, mean (SD) & $2.8(0.6)$ & $2.9(0.5)$ & 0.59 \\
\hline TLC, / $/ \mu \mathrm{L}$, mean (SD) & $1426(793)$ & $1555(751)$ & 0.29 \\
\hline C-reactive protein, mg/dL, mean (SD) & $2.1(2.6)$ & $2.5(3.3)$ & 0.99 \\
\hline Total cholesterol, mg/dL, mean (SD) & $151(44)$ & $150(31)$ & 0.95 \\
\hline Blood urea nitrogen, $\mathrm{mg} / \mathrm{dL}$, mean (SD) & $24.8(18.3)$ & $22.5(13.0)$ & 0.54 \\
\hline PT-INR, mean (SD) & $1.21(0.23)$ & $1.14(0.10)$ & 0.02 \\
\hline
\end{tabular}

SD, standard deviation; TLC, total lymphocyte count; PT-INR, international normalized ratio of prothrombin time.

orders, diabetes mellitus, pressure ulcers and malignancy between both groups, there were significantly more patients with dementia in the Push kit group when compared to the Introducer kit group ( $44 \%$ vs. $21.3 \%, P<0.01$ ). As for preoperative biomarkers, there were no differences in body mass indices (BMI), white blood cell counts, hemoglobin levels, platelet counts, serum albumin levels, total lymphocyte counts, C-reactive protein levels, total cholesterol levels and blood urea nitrogen levels between the 2 groups. Although the use of antithrombotic therapy did not differ significantly between both groups, patients in the Push kit group had a higher international normalized ratio of prothrombin time (PT-INR) compared to those in the Introducer kit group (1.21 vs. $1.14, P=0.02$ ). Indications for PEG were primarily dysphagia secondary to neurological impairments or insufficient oral intake which may be due to various underlying conditions as listed in the comorbidities section ( $\bullet$ Table 1 ).

Tube placements were successful in all patients for both PEG kits and hemodynamic monitoring during procedure is summarized in Table 2. Conscious sedation with diazepam was used in almost $75 \%$ of patients in the Push kit group, which was significantly higher than the $57 \%$ of patients in the Introducer kit group $(P=0.03)$. However, when used, the mean diazepam dosage did not differ significantly between the 2 groups. There were no significant differences in mean arterial pressure (MAP), pulse rate and saturation of peripheral oxygen $\left(\mathrm{SpO}^{2}\right)$ at baseline when comparing both groups. Postoperative changes (the difference between values taken immediately after procedure and baseline) in hemodynamic markers were also insignificant between both groups although it should be noted that the increase in mean MAP for the Introducer kit group was slightly more prominent. Laboratory biomarkers were collected on postoperative Day 7 according to protocol and the differences from preoperative values are shown in Table 3 . The changes in hemoglobin levels, serum albumin levels, blood urea nitrogen as well as inflammatory mar- kers such as white blood count and C-reactive protein levels were similar no matter which PEG kit was used.

- Table 4 summarizes the postoperative adverse events and clinical outcomes after PEG tube placement. Feeding-related aspiration pneumonia (established by clinical symptoms as well as radiological findings) was the most common adverse event, followed by peristomal infection and feeding-related diarrhea. There were significantly fewer occurrences of aspiration pneumonia in the Introducer kit group compared to the Push kit group ( $8.2 \%$ vs. $24 \%, P=0.02$ ) and although not statistically significant, fewer cases of feeding-related diarrhea in the Introducer kit group as well. The frequency of peristomal infection was similar in both groups but the average Jain's infection score of patients in the Introducer kit group were significantly lower compared to the Push kit group (1.2 vs. 1.6, $P<0.01$ ). There were no significant differences in terms of accidental tube dislodgements, peritonitis and bleeding that required intervention (such as blood transfusion). No tube obstruction was observed in either groups during postoperative clinical course (up to day 90).

Postoperative length of stay was significantly shorter in the Introducer kit group (16 days vs. 23.7 days, $P=0.01$ ). There were also fewer mortalities (in-hospital, 14-day, 30-day, 60-day and 90-day) in the Introducer kit group with the 60-day mortality being significantly lower compared to the Push kit group (3.3\% vs. $16 \%, P=0.02$ ). There was one procedure-related death in the Push kit group (due to excessive bleeding) but the main contributor to all mortality was aspiration pneumonia secondary to gastroesophageal reflux of feed. 
Table 2 Hemodynamic monitoring during gastrostomy procedure.

\begin{tabular}{|c|c|c|c|}
\hline & $\begin{array}{l}\text { Push kit } \\
(n=75)\end{array}$ & $\begin{array}{l}\text { Introducer kit } \\
(n=61)\end{array}$ & $P$ value \\
\hline Use of sedation with diazepam, $\mathrm{n}(\%)$ & $56(74.7)$ & $35(57.4)$ & 0.03 \\
\hline Mean diazepam dosage used, $\mathrm{mg}$ & $4.2(1.9)$ & $3.9(2.1)$ & 0.25 \\
\hline \multicolumn{4}{|l|}{ Baseline } \\
\hline MAP, $\mathrm{mmHg}$ & $93.1(15.5)$ & $93.1(18.7)$ & 0.61 \\
\hline Pulse rate, beats per minute & $81.6(15.7)$ & $77.9(13.4)$ & 0.16 \\
\hline $\mathrm{SpO}^{2}, \%$ & $97.4(2.3)$ & $97.7(2.7)$ & 0.32 \\
\hline \multicolumn{4}{|c|}{ Postoperative change (immediately after procedure) } \\
\hline$\triangle \mathrm{MAP}, \mathrm{mmHg}$ & $1.8(18.9)$ & $8.1(19.4)$ & 0.07 \\
\hline$\triangle$ Pulse rate, beats per minute & $4.6(15.7)$ & $4.9(11.1)$ & 0.71 \\
\hline$\triangle \mathrm{SpO}^{2}, \%$ & $-0.5(2.8)$ & $0.0(2.6)$ & 0.69 \\
\hline
\end{tabular}

Values expressed as mean (SD) unless otherwise specified. MAP, mean arterial pressure; $\mathrm{SpO}^{2}$, saturation of peripheral oxygen.

Table 3 Postoperative (at 7 day) change in laboratory biomarkers.

\begin{tabular}{|llll} 
& $\begin{array}{l}\text { Push kit } \\
(\mathbf{n = 7 5 )}\end{array}$ & $\begin{array}{l}\text { Introducer kit } \\
(\mathbf{n = 6 1 )}\end{array}$ & P value \\
\hline$\Delta$ White blood count, $/ \mu \mathrm{L}$ & $212(3443)$ & $813(2839)$ & 0.26 \\
\hline$\Delta$ Hemoglobin, g/dL & $-0.5(0.9)$ & $-0.6(1.2)$ & 0.84 \\
\hline$\Delta$-reactive protein, $\mathrm{mg} / \mathrm{dL}$ & $0.4(3.2)$ & $0.1(4.1)$ & 0.45 \\
\hline$\Delta$ Serum albumin, $\mathrm{g} / \mathrm{dL}$ & $-0.1(0.3)$ & $-0.1(0.3)$ & 0.70 \\
\hline$\Delta$ Blood urea nitrogen, $\mathrm{mg} / \mathrm{dL}$ & $-0.9(14.7)$ & $0.6(6.9)$ & 0.88 \\
\hline
\end{tabular}

Values expressed as mean (SD).

Table 4 Postoperative adverse events and clinical outcomes

\begin{tabular}{|c|c|c|c|}
\hline & $\begin{array}{l}\text { Push kit } \\
(n=75)\end{array}$ & $\begin{array}{l}\text { Introducer kit } \\
(n=61)\end{array}$ & $P$ value \\
\hline \multicolumn{4}{|l|}{ Postoperative adverse events } \\
\hline Feeding-related aspiration pneumonia & $18(24)$ & $5(8.2)$ & 0.02 \\
\hline Peristomal infection & $10(13.3)$ & $7(11.5)$ & 0.75 \\
\hline Jain's infection score, mean (SD) & $1.6(1.5)$ & $1.2(1.5)$ & $<0.01$ \\
\hline Feeding-related diarrhea & $10(13.3)$ & $2(3.3)$ & 0.06 \\
\hline Accidental tube dislodgement & $1(1.3)$ & $2(3.3)$ & 0.59 \\
\hline Peritonitis & $1(1.3)$ & $1(1.6)$ & 1.00 \\
\hline Bleeding & $3(4)$ & $1(1.6)$ & 0.63 \\
\hline Tube obstruction & $0(0)$ & $0(0)$ & $\mathrm{N} / \mathrm{A}$ \\
\hline \multicolumn{4}{|l|}{ Clinical outcomes } \\
\hline Length of stay, days, mean (SD) & $23.7(21.2)$ & $16.0(13.9)$ & 0.01 \\
\hline In-hospital mortality & $11(14.7)$ & $3(4.9)$ & 0.06 \\
\hline 14-day mortality & $4(5.3)$ & $1(1.6)$ & 0.38 \\
\hline 30-day mortality & $7(9.3)$ & $1(1.6)$ & 0.07 \\
\hline 60-day mortality & $12(16)$ & $2(3.3)$ & 0.02 \\
\hline 90-day mortality & $15(20)$ & $5(8.2)$ & 0.05 \\
\hline Procedure-related mortality & $1(1.3)$ & $0(0)$ & 1.00 \\
\hline
\end{tabular}

Values expressed as n (\%) unless otherwise specified. N/A: Not applicable.

\section{Discussion}

For long-term enteral tube feeding, PEG has been established as a safe and simple procedure. Although the pull or push technique remains the most popular method for gastrostomy tube placement, the need of passing the tube through the oral cavity increases the risk of peristomal site infection and negatively affects the success rate of tube placement in patients with upper digestive tract stenosis or trismus.

PEG using the introducer technique is an alternative method for gastrostomy tube placement which does not require tube transit orally. Unlike the pull or push technique, the procedure requires only single insertion of the endoscope and the endoscopist is required to just observe during insertion. However due to several reasons, this method of tube placement is not as widely employed as the pull or push technique. First of all, initial versions of the introducer technique did not use gastropexy, which while making the procedure simpler and faster, also caused adverse events such as displacement of the gastric wall due to trocar insertion or accidental tube dislodgement, which in turn resulted in leakage of stomach contents into the peritoneum and procedural failure $[22,23]$. The introduction of gastropexy before tube 
placement greatly enhanced the safety of the introducer technique $[24,25]$. However, the need for gastropexy and gastropexy-related devices not only increased total procedure time but also the price of introducer PEG kits [17,26,27]. Furthermore, almost all commercially available kits use small-caliber gastrostomy tubes of up to $15 \mathrm{Fr}$, which may lead to frequent episodes of tube obstruction. Tube size is also a matter of concern in Japan where the use of semi-solid feed or blended food is quite common to help prevent gastroesophageal reflux and other feedingrelated adverse events $[28,29]$.

The modified introducer technique (also called the direct method) was developed in 2001 and enabled the placement of largecaliber gastrostomy tubes up to $24 \mathrm{Fr}[30,31]$. However, this modification actually made the procedure more complex due to the need of puncture site dilation. Prices of modified introducer PEG kits are also more expensive than the original introducer PEG kits. Recently however, an introducer technique using a new large-caliber trocar to insert gastrostomy tubes up to $20 \mathrm{Fr}$ was reported [32]. Although the trocar size is similar to the one used in this study, it lacked the safety features of the sleeve protection system described herein.

This is the first study evaluating the safety and utility of a recently developed large-caliber (20 Fr) introducer PEG kit with an improved puncture needle using a sleeve protection system. As far as we are aware, this is also the first study comparing the introducer technique and the push technique using gastrostomy tubes of the same size. Although this is a retrospective non-randomized study, other than the prevalence of dementia, patients in both groups were comparable in terms of baseline characteristics such as age, gender and other comorbidities. Preoperative biomarkers also did not differ significantly in both groups except for PT-INR, which was slightly higher in the Push kit group.Although the higher PT-INR may have indirectly contributed to more incidence of bleeding (including 1 procedure-related death) in the Push kit group, it should be noted that the average values for both groups were well within guideline-acceptable levels. As shown in $\bullet$ Table 3, postoperative changes in hemoglobin levels on Day 7 also did not differ significantly between the 2 groups, although the use of blood transfusion was not taken into consideration during analysis.

In this study, postoperative changes in data were used to reflect the changes from baseline, meaning that a positive value would indicate an increase and vice versa. Hemodynamic changes during procedure ( $\bullet$ Table 2$)$ and postoperative changes in laboratory biomarkers 7 days after procedure $(\bullet$ Table 3 ) indicate that the use of the introducer PEG kit was well tolerated by patients. The use of sedation (not the dosage however) was significantly lower in the Introducer kit group presumably because only single insertion of endoscope was required. This may have influenced the postoperative changes in hemodynamics such as MAP, albeit not to a statistically significant degree when compared to the Push kit group.

As for postoperative adverse events, the Introducer kit group fared better when it came to feeding-related events such as aspiration pneumonia and diarrhea (for diarrhea, not statistically significant) despite both groups being similar in terms of preoperative nutritional biomarkers and having the same feeding protocols. However, because patients in this study were not randomized accordingly, these favorable outcomes could have resulted from selection bias. Although the occurrence of peristomal infection did not differ significantly between the two groups, in agreement with earlier studies, patients in the Introducer kit group had significantly lower infection scores compared to the Push kit group. Previous studies have also reported higher rates of accidental tube dislodgement (removal) in patients receiving tube placement using the introducer technique $[19,33]$. Although we contend that the higher rates may be due to the type of gastrostomy tube (balloon-type) employed rather than the insertion technique itself, in this study, tube dislodgements were not significantly higher in the Introducer kit group. Interestingly, the incidence of bleeding was also not higher in the Introducer kit group although gastropexy (meaning more percutaneous gastric punctures) was required.

Postoperative length of stay was significantly lower and there were fewer mortalities in the Introducer kit group, with statistical significance at the 60-day mortality point. This is most probably because patients in the Introducer kit group experienced fewer adverse events compared to their Push kit group counterparts. Notable limitations of this study include its retrospective design with a high probability of selection bias even though baseline characteristics and biomarkers were reasonably comparable between the 2 groups. The study also lacks long term follow-up data beyond 90 days (which is still in progress as an ongoing observational study).

It may be worth mentioning that in Japan, regular gastrostomy tube replacement (every 3 to 4 months) is common to prevent tube obstruction and other long-term tube-related problems. Gastrostomy tubes placed using the pull or push techniques usually have internal mushroom-like bumpers which often require endoscopic removal for optimal safety during replacement while tubes placed using the introducer technique uses retention balloons which upon deflation can be safely and easily replaced without endoscopic intervention. Therefore, using the introducer PEG kits may also help reduce the cost of initial tube replacements.

\section{Conclusions}

\section{$\nabla$}

In conclusion, retrospective evaluation of the safety and utility of a novel large-caliber introducer PEG kit with an improvised puncture needle/trocar showed that gastrostomy tubes placed using the new kit produced non-inferior (with some favourable) results when compared to push PEG kits of similar tube size. We also contend that the new kit would be very useful, especially in patients with esophageal stenosis, since using the large-caliber tubes will help maintain the same tube patency as tubes inserted using the conventional pull/push techniques. Balloon type catheters also allow tube exchange without the need for an endoscopic examination. Although the new introducer PEG kit is considered safe to use, the final choice of gastrostomy tube placement method should be made after taking into consideration patient's circumstances (giving priority to cases with trismus or upper digestive tract stenosis) as well as operator's technical preference.

\section{Competing interests: None}

\section{Acknowledgments \\ $\nabla$}

CREATE MEDIC Co., Ltd provided the unannotated pictures for - Fig. 1 and $\odot$ Fig. 2. 


\section{References}

1 Gauderer MW, Ponsky JL, Izant RJJr. Gastrostomy without laparotomy: a percutaneous endoscopic technique. J Pediatr Surg 1980; 15: 872 - 875

2 DeLegge MH. Percutaneous endoscopic gastrostomy. Am J Gastroenterol 2007; 102: 2620-2623

3 Suzuki Y, Tamez S, Murakami A et al. Survival of geriatric patients after percutaneous endoscopic gastrostomy in Japan. World J Gastroenterol 2010; 16: $5084-5091$

4 Richter-Schrag HJ, Richter S, Ruthmann $\mathrm{O}$ et al. Risk factors and complications following percutaneous endoscopic gastrostomy: a case series of 1041 patients. Can J Gastroenterol 2011; 25: 201 - 206

5 Gossner L, Keymling J, Hahn EG et al. Antibiotic prophylaxis in percutaneous endoscopic gastrostomy (PEG): a prospective randomized clinical trial. Endoscopy 1999; 31: 119-124

6 Lipp A, Lusardi G. Systemic antimicrobial prophylaxis for percutaneous endoscopic gastrostomy. Cochrane Database Syst Rev 2013; 11: CD005571

7 Lee C, Im JP, Kim JW et al. Risk factors for complications and mortality of percutaneous endoscopic gastrostomy: a multicenter, retrospective study. Surg Endosc 2013; 27: 3806-3815

8 Wirth R, Bauer J, Sieber C. Necrotizing Candida infection after percutaneous endoscopic gastrostomy: a fatal and rare complication. JPEN J Parenter Enteral Nutr 2008; 32: 285-287

9 Hashiba K. Technic for opening a gastrostomy under endoscopic control and manipulation. Rev Paul Med 1980; 95: 37- 38 [article in portuguese]

10 Ueno F, Kadota T. Percutaneous endoscopic gastrostomy- Report of a simplified new technique. Progress of Digestive Endoscopy 1983; 23 : 60-62 [article in japanese]

11 Russell TR, Brotman M, Norris F. Percutaneous gastrostomy. A new simplified and cost-effective technique. Am J Surg 1984; 148: 132 - 137

12 Foster JM, Filocamo P, Nava $\mathrm{H}$ et al. The introducer technique is the optimal method for placing percutaneous endoscopic gastrostomy tubes in head and neck cancer patients. Surg Endosc 2007; 21: 897-901

13 Riley DA, Strauss M. Airway and other complications of percutaneous endoscopic gastrostomy in head and neck cancer patients. Ann Otol Rhinol Laryngol 1992; 101: 310-313

14 Huang AT, Georgolios A, Espino $S$ et al. Percutaneous endoscopic gastrostomy site metastasis from head and neck squamous cell carcinoma: case series and literature review. J Otolaryngol Head Neck Surg 2013; $42: 20$

15 Maetani I, Tada T, Ukita T et al. PEG with introducer or pull method: a prospective randomized comparison. Gastrointest Endosc 2003; 57: $837-841$

16 Campoli PM, de Paula AA, Alves LG et al. Effect of the introducer technique compared with the pull technique on the peristomal infection rate in PEG: a meta-analysis. Gastrointest Endosc 2012; 75: 988-996

17 Shastri YM, Hoepffner N, Tessmer A et al. New introducer PEG gastropexy does not require prophylactic antibiotics: multicenter prospec- tive randomized double-blind placebo-controlled study. Gastrointest Endosc 2008; 67: 620-628

18 Loser C, Aschl G, Hébuterne X et al. ESPEN guidelines on artificial enteral nutrition: percutaneous endoscopic gastrostomy (PEG). Clin Nutr 2005; $24: 848-861$

19 Lee SW, Lee JH, Cho H et al. Comparison of clinical outcomes associated with pull-type and introducer-type percutaneous endoscopic gastrostomies. Clin Endosc 2014; 47: 530-537

20 Sacks BA, Vine HS, Palestrant AM et al. A nonoperative technique for establishment of a gastrostomy in the dog. Invest Radiol 1983; 18: 485487

21 Jain $N K$, Larson $D E$, Schroeder $K W$ et al. Antibiotic prophylaxis for percutaneous endoscopic gastrostomy. A prospective, randomized, double-blind clinical trial. Ann Intern Med 1987; 107: 824-828

22 Miller RE, Kummer BA, Tiszenkel HI et al. Percutaneous endoscopic gastrostomy. Procedure of choice. Ann Surg 1986; 204: 543-545

23 Kozarek RA, Ball TJ, Ryan JAJr. When push comes to shove: a comparison between two methods of percutaneous endoscopic gastrostomy. Am J Gastroenterol 1986; 81: 642 - 646

24 Hashiba K. Endoscopic gastrostomy. Endoscopy 1987; 19: 23-24

25 Dormann AJ, Glosemeyer R, Leistner U et al. Modified percutaneous endoscopic gastrostomy (PEG) with gastropexy-early experience with a new introducer technique. Z Gastroenterol 2000; 38: 933-938

26 Martins FP, Sousa MC, Ferrari AP. New "introducer" PEG-gastropexy with T fasteners: a pilot study. Arq Gastroenterol 2011; 48: 231-235

27 Okumura N, Tsuji N, Ozaki $N$ et al. Percutaneous endoscopic gastrostomy with Funada-style gastropexy greatly reduces the risk of peristomal infection. Gastroenterol Rep (Oxf) 2015; 3: 69-74

28 Kanie J, Suzuki Y, Iguchi A et al. Prevention of gastroesophageal reflux using an application of half-solid nutrients in patients with percutaneous endoscopic gastrostomy feeding. J Am Geriatr Soc 2004; 52: $466-467$

29 Nishiwaki S, Araki H, Shirakami Y et al. Inhibition of gastroesophageal reflux by semi-solid nutrients in patients with percutaneous endoscopic gastrostomy. JPEN J Parenter Enteral Nutr 2009; 33: 513-519

30 Horiuchi A, Nakayama $Y$, Tanaka $N$ et al. Prospective randomized trial comparing the direct method using a $24 \mathrm{Fr}$ bumper-button-type device with the pull method for percutaneous endoscopic gastrostomy. Endoscopy 2008; 40: $722-726$

31 Shigoka H, Maetani I, Tominaga K et al. Comparison of modified introducer method with pull method for percutaneous endoscopic gastrostomy: prospective randomized study. Dig Endosc 2012; 24: 426-431

32 Giordano-Nappi JH, Maluf-Filho F, Ishioka $S$ et al. A new large-caliber trocar for percutaneous endoscopic gastrostomy by the introducer technique in head and neck cancer patients. Endoscopy 2011; 43: $752-758$

33 Van Dyck E, Macken EJ, Roth B et al. Safety of pull-type and introducer percutaneous endoscopic gastrostomy tubes in oncology patients: a retrospective analysis. BMC Gastroenterol 2011; 11: 23 